\title{
Number Theoretic Study in Quantum Interactions
}

\author{
Masato Wakayama
}

\begin{abstract}
The quantum interaction models, with the quantum Rabi model as a distinguished representative, are recently appearing ubiquitously in various quantum systems including cavity and circuit quantum electrodynamics, quantum dots and artificial atoms, with potential applications in quantum information technologies including quantum cryptography and quantum computing (Haroche and Raimond 2008; Yoshihara et al. 2018). In this extended abstract, based on the contents of the talk at the conference, we describe shortly certain number theoretical aspects arising from the non-commutative harmonic oscillators (NCHO: see Parmeggiani and Wakayama 2001; Parmeggiani 2010) and quantum Rabi model (QRM: see Braak 2011 for the integrability) through their respective spectral zeta functions.
\end{abstract}

The quantum interaction models, with the quantum Rabi model as a distinguished representative, are recently appearing ubiquitously in various quantum systems including cavity and circuit quantum electrodynamics, quantum dots and artificial atoms, with potential applications in quantum information technologies including quantum cryptography and quantum computing (Haroche and Raimond 2008; Yoshihara et al. 2018). In this extended abstract, based on the contents of the talk at the conference, we describe shortly certain number theoretical aspects arising from the non-commutative harmonic oscillators (NCHO: see Parmeggiani and Wakayama 2001; Parmeggiani 2010) and quantum Rabi model (QRM: see Braak 2011 for the integrability) through their respective spectral zeta functions.

In physics, given a quantum interaction model, one of the main interests is to know the heat kernel (or equivalently the evolution operator) since, among other reasons, the heat kernel gives the partition function by taking the trace. With partition function of the model, we may also get the analytic properties of the spectral zeta function

\footnotetext{
M. Wakayama (凶)

Department of Mathematics, Tokyo University of Science, 1-3 Kagura-zaka, Shinjyuku-ku,

Tokyo 162-8601, Japan

e-mail:wakayama@rs.tus.ac.jp; wakayama@imi.kyushu-u.ac.jp

Institute of Mathematics for Industry, Kyushu University, 744 Motooka, Nishi-ku Fukuoka 819-0395, Japan
}

T. Takagi et al. (eds.), International Symposium on Mathematics, Quantum Theory, and Cryptography, Mathematics for Industry 33, https://doi.org/10.1007/978-981-15-5191-8_10 
by means of the Mellin transform. A spectral zeta function is defined, in general, as the Dirichlet series formed by the spectrum (eigenvalues) of the corresponding Hamiltonian (Ichinose and Wakayama 2005; Sugiyama 2018). Notice that knowing the spectral zeta function is essentially equivalent to knowing the partition function in any quantum system.

In the case of the $\mathrm{NCHO}$, the Hamiltonian is given by

$$
Q=\left(\begin{array}{ll}
\alpha & 0 \\
0 & \beta
\end{array}\right)\left(-\frac{1}{2} \frac{d^{2}}{d x^{2}}+\frac{1}{2} x^{2}\right)+\left(\begin{array}{cc}
0 & -1 \\
1 & 0
\end{array}\right)\left(x \frac{d}{d x}+\frac{1}{2}\right),
$$

with $\alpha, \beta>0$ and $\alpha \beta>1$ (the condition for having only a discrete spectrum with positive eigenvalues), and the spectral zeta function by

$$
\zeta_{Q}(s):=\sum_{n=1}^{\infty} \lambda_{n}^{-s}(\Re(s)>1),
$$

where $(0<) \lambda_{1}<\lambda_{2} \leq \lambda_{3} \leq \ldots(\nearrow \infty)$ are the eigenvalues of NCHO. Note that the lowest eigenstate is multiplicity free (Hiroshima and Sasaki 2014) and the multiplicity of general eigenstate is less than or equal to two (Wakayama 2016). The function $\zeta_{Q}(s)$ is meromorphically continued to the whole complex plane with a unique simple pole at $s=1$ and has trivial zeros at the even non-positive integers (Ichinose and Wakayama 2005). Although our study is very much influenced by the classical algebro-geometric work on Apéry numbers for the Riemann zeta function in Beukers (1987) and its subsequent developments, since the family of generating functions for Apéry-like numbers (Kimoto and Wakayama 2006) arising via the NCHO possesses a remarkable hierarchical structure, there is a decisive difference between these two (Ichinose and Wakayama 2005; Kimoto and Wakayama 2019).

For instance, there are congruence properties of the (normalized) Apéry-like numbers that have arisen naturally from the special values $\zeta_{Q}(2)$ at $s=2$. This can be seen by the same idea that guided the studies for the Apéry numbers for $\zeta(2)\left(=\pi^{2} / 6\right)$ in Beukers (1985). These congruence properties led us further to observe that the generating function $w_{2}$ of the Apéry-like numbers for $\zeta_{Q}(2)$ is interpreted as a $\Gamma(2)$ modular form of weight 1 (Kimoto and Wakayama 2007) in the same way as in a pioneering study by Beukers $(1983,1987)$ for the Apéry numbers. It is worth mentioning that the recurrence equation of these Apéry-like numbers defined in Kimoto and Wakayama (2006) provides one of the particular examples listed in Zagier (2009) (it gives \#19 in the list). ${ }^{1}$ Also, recently, certain congruence relations among these Apéry-like numbers conjectured in Kimoto and Wakayama (2006) resembling Rodriguez-Villegas type congruences (Mortenson 2003) were proved in Long et al. (2016). It is, however, hard in general to obtain precise information, in the same level of $\zeta_{Q}(2)$, of the higher special values of $\zeta_{Q}(n)(n>2)$. Thus, we introduce the Apéry-like numbers $J_{k}(n)(k=0,1,2, \ldots)$ for each $n$ defined through

\footnotetext{
${ }^{1}$ Although the terminology "Apéry-like" is the identical, the usage/definition of the name in the current paper is different from the one in the title of Zagier (2009).
} 
the first anomaly of $\zeta_{Q}(n)(n>2)$ (Kimoto and Wakayama 2019) (see also Kimoto (2016)). These Apéry-like numbers share the properties of the one for $\zeta_{Q}(2)$, e.g. satisfy a similar recurrence relation as in the case of $\zeta_{Q}(2)$ and hence the ordinary differential equation satisfied by the generating function follows from the recurrence relation. Remarkably, the homogeneous part of each of the differential equations is identified with a ( $n$ dependent) power of the homogeneous part of the one corresponding to $\zeta_{Q}(2)$. Further, we observe that the meta-generating functions of Apéry-like numbers $J_{k}(n)$ are described explicitly by the modular Mahler measures studied by Rodriguez-Villegas in Rodriguez (1999). Through this relation, we may find an interesting aspect of a discrete dynamical system behind NCHO defined by a certain limit of finite abelian group via (weighted) Cayley graphs studied in Dasbach and Lalin (2009). Moreover, we note here (Kimoto and Wakayama 2012, 2019) that the generating function $w_{2 n}$ of Apéry-like numbers corresponding to the first anomaly in $\zeta_{Q}(2 n)$ when $n=2$ is given by an automorphic integral with a rational period function in the sense of Knopp (1978). This is obviously a generalization of our earlier result (Kimoto and Wakayama 2007) showing that $w_{2}$ is interpreted as a $\Gamma(2)$-modular form of weight 1.

Furthermore, we show certain congruence relations among these normalized Apéry-like numbers which are the generalization of the results in Kimoto and Wakayama (2006). A possible generalization of the results in Liu (2018) seems very interesting. We also conjecture much stronger results based on numerical experiments in Kimoto and Wakayama (2019).

The Hamiltonian $H_{\text {Rabi }}$ of the QRM is precisely given by

$$
H_{\text {Rabi }}:=\omega a^{\dagger} a+\Delta \sigma_{z}+g\left(a+a^{\dagger}\right) \sigma_{x} .
$$

Here, $a^{\dagger}$ and $a$ are the creation and annihilation operators of the single bosonic mode $\left(\left[a, a^{\dagger}\right]=1\right), \sigma_{x}, \sigma_{z}$ are the Pauli matrices (sometimes written as $\sigma_{1}$ and $\sigma_{3}$, but since there is no risk of confusion with the variable $x$ to appear below in the heat kernel, we use the usual notations), $2 \Delta$ is the energy difference between the two levels, and $g$ denotes the coupling strength between the two-level system and the bosonic mode with frequency $\omega$ (subsequently, we set $\omega=1$ without loss of generality). The integrability of the QRM was established in Braak (2011) using the well-known $\mathbb{Z}_{2}$-symmetry of the Hamiltonian $H_{\text {Rabi }}$, usually called parity.

In the case of QRM, we recently obtained the (analytic formula of) heat kernel (Reyes and Wakayama 2019) using the Trotter-Kato product formula by extensive discussions of combinatorics and graph theory including quantum Fourier transform.

Concretely, the heat kernel $K_{\mathrm{Rabi}}(t, x, y)$ of the QRM is given by

$$
K_{\mathrm{Rabi}}(t, x, y)=\widetilde{K}_{0}(x, y, g, t) \sum_{\lambda=0}^{\infty}(t \Delta)^{\lambda} \Phi_{\lambda}(x, y, g, t) .
$$

Here the $2 \times 2$ matrix-valued function $\Phi_{\lambda}(g, t)$ for $\lambda \geq 0$ is given by 


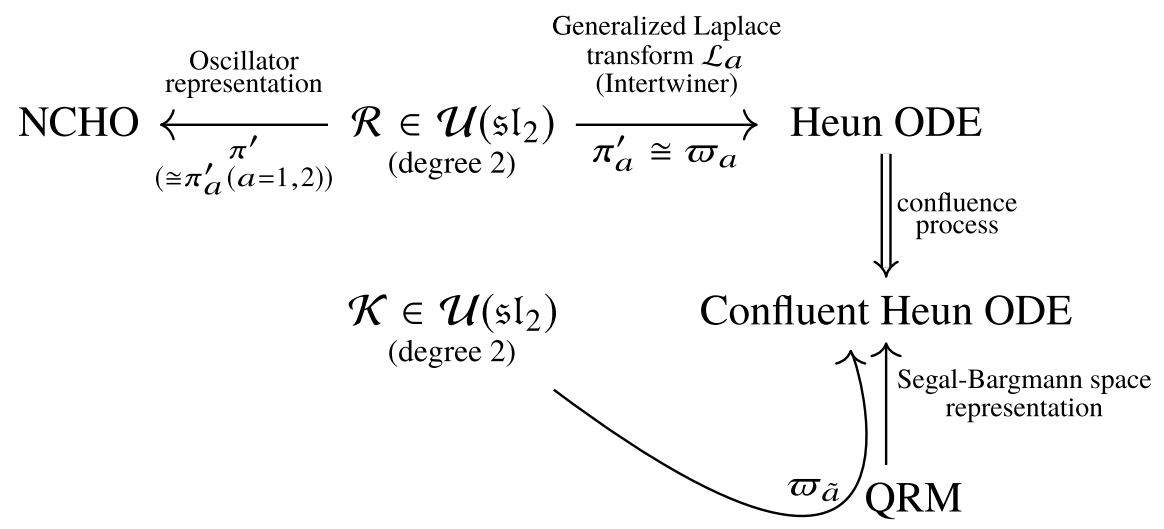

Fig. 1 From the NCHO to QRM (Heun's Pictures)

$$
\begin{aligned}
\Phi_{\lambda}(x, y, g, t)=\int_{0 \leq \mu_{1} \leq \cdots \leq \mu_{\lambda} \leq 1} \cdots \int^{\phi\left(\mu_{\lambda}, t\right)+\xi_{\lambda}\left(\boldsymbol{\mu}_{\lambda}, t\right)} & {\left[\begin{array}{c}
(-1)^{\lambda} \cosh (-1)^{\lambda+1} \sinh \\
-\sinh \quad \cosh
\end{array}\right] } \\
& \times\left(\theta_{\lambda}\left(x, y, \boldsymbol{\mu}_{\lambda}, t\right)\right) d \boldsymbol{\mu}_{\lambda}
\end{aligned}
$$

where $\boldsymbol{\mu}_{\lambda}=\left(\mu_{1}, \mu_{2}, \cdots, \mu_{\lambda}\right)$ and $d \boldsymbol{\mu}_{\lambda}=d \mu_{1} d \mu_{2} \cdots d \mu_{\lambda}$ with $\boldsymbol{\mu}_{\mathbf{0}}=0$ and $d \boldsymbol{\mu}_{\mathbf{0}}=$ 1. For the definition of the functions $\phi, \xi_{\lambda}, \theta_{\lambda}$ and $\widetilde{K}_{0}$, (Mehler's kernel) the reader is directed to Reyes and Wakayama (2019).

This is the first time an explicit determination of the heat kernel is obtained for an interacting system (though certain partial results have been discussed, e.g. in Legget 1987 for the Spin-Boson model and Anderson et al. 1970; Chakravarty 1995 for the Kondo effect using the Feynman-Kac formula.) The heat kernel formula allows us to have the contour integral representation of the spectral zeta function of the QRM (Sugiyama 2018) and open the study of the special values of negative integral points using it (Reyes and Wakayama 2019).

Further, although NCHO is not confirmed as a practical physical model, it may be considered as a "covering" model of QRM through the respective Heun ODE pictures (Wakayama 2016) (Fig. 1). Thus, in addition to the study of the respective number theoretical aspects of the models independently, the comparison of the number theoretic objects appearing from each model is an interesting and significant problem.

In addition to the number theoretic structure described above, we remark here that there appear certain algebraic curves, including elliptic and super elliptic curves, in the description of degenerations of the eigenstates for the asymmetric QRM with an integral perturbation parameter (Wakayama 2017; Kimoto et al. 2020; Reyes and Wakayama 2017). This shows another mathematical structure behind the asymmetric and symmetric QRM.

The following figure (Fig. 2) illustrates the position of this extended abstract from our whole interest. Particularly, the talk focused on the special values of such zeta 


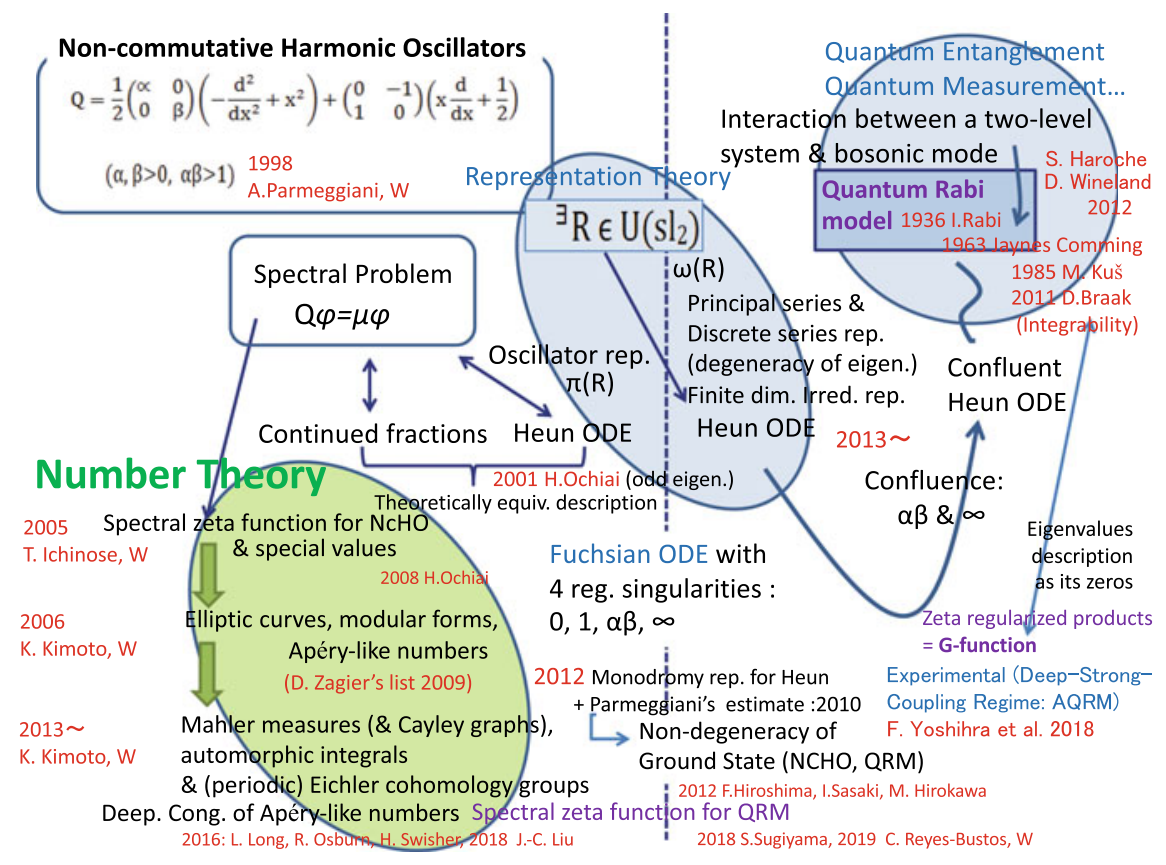

Fig. 2 Non-commutative harmonic oscillator and (asymmetric and symmetric) quantum Rabi models

functions (Ichinose and Wakayama 2005; Ochiai 2008; Kimoto and Wakayama 2006, 2007, 2012; Long et al. 2016; Liu 2018; Kimoto and Wakayama 2019). We note that special values of zetas may be considered as the moments of the partition function of the corresponding model.

\section{References}

P.W. Anderson, G. Yuval, D.R. Hamann, Exact results in the kondo problem. II. Scaling theory, qualitatively correct solution, and some new results on one-dimensional classical statistical models. Phys. Rev. B 1, 4464 (1970)

F. Beukers, Irrationality of $\pi^{2}$, periods of an elliptic curve and $\Gamma_{1}(5)$, Diophantine approximations and transcendental numbers (Luminy, 1982). Progr. Math. 31, 47-66, Birkhäuser, Boston (1983)

F. Beukers, Some congruences for the Apéry numbers. J. Number Theo. 21, 141-155 (1985)

F. Beukers, Irrationality proofs using modular forms. Soc. Math. France, Astérisque 21, 271-283 (1987)

D. Braak, Integrability of the Rabi model. Phys. Rev. Lett. 107, 100401 (2011)

S. Chakravarty, J. Rudnick, Dissipative dynamics of a two-state system, the Kondo problem, and the inverse-square Ising model. Phys. Rev. Lett. 75, 501 (1995)

O. Dasbach, M. Lalin, Mahler measure under variations of the base group. Forum Math. 21, 621-637 (2009) 
S. Haroche, J.M. Raimond, Exploring the Quantum - Atoms Cavities and Photons (Oxford University Press, Oxford, 2008)

F. Hiroshima, I. Sasaki, Spectral analysis of non-commutative harmonic oscillators: the lowest eigenvalue and no crossing. J. Math. Anal. Appl. 105, 595-609 (2014)

T. Ichinose, M. Wakayama, Zeta functions for the spectrum of the non-commutative harmonic oscillators. Commun. Math. Phys. 258, 697-739 (2005)

T. Ichinose, M. Wakayama, Special values of the spectral zeta function of the non-commutative harmonic oscillator and confluent Heun equations. Kyushu J. Math. 59, 39-100 (2005)

K. Kimoto, Generalized Apéry numbers arising from the non-commutative harmonic oscillator. Ryukyu Math. J. 29, 1-31 (2016)

K. Kimoto, C. Reyes-Bustos, M. Wakayama, Determinant expressions of constraint polynomials and degeneracies of the asymmetric quantum Rabi model. Int. Math. Res. Notices (2020), Published online 20, April, arXiv:1712.04152

K. Kimoto, M. Wakayama, Apéry-like numbers arising from special values of spectral zeta functions for non-commutative harmonic oscillators. Kyushu J. Math. 60, 383-404 (2006)

K. Kimoto, M. Wakayama, Elliptic curves arising from the spectral zeta function for noncommutative harmonic oscillators and $\Gamma_{0}(4)$ - modular forms, in Proceedings Conference on L-Functions, eds. by L. Weng, M. Kaneko, pp. 201-218. World Scientific (2007)

K. Kimoto, M. Wakayama, Spectrum of non-commutative harmonic oscillators and residual modular forms, in Noncommutative Geometry and Physics, eds. by G. Dito, et al (World Scientific, Singapore, 2012), pp. 237-267

K. Kimoto, M. Wakayama, Apéry-like numbers for non-commutative harmonic oscillators and automorphic integrals, http://arxiv.org/abs/1905.01775

I. Knopp, Rational period functions of the modular group. With an appendix by Georges Grinstein. Duke Math. J. 45, 47-62 (1978)

A.J. Legget et al., Dynamics of the dissipative two-state system. Rev. Mod. Phys. 59, 1-85 (1987)

J.-C. Liu, A generalized supercongruence of Kimoto and Wakayama. J. Math. Anal. Appl. 467, $15-25$ (2018)

L. Long, R. Osburn, H. Swisher, On a conjecture of Kimoto and Wakayama. Proc. Amer. Math. Soc. 144, 4319-4327 (2016)

E. Mortenson, A supercongruence conjecture of Rodriguez-Villegas for a certain truncated hypergeometric function. J. Number Theo. 99, 139-147 (2003)

$\mathrm{H}$. Ochiai, A special value of the spectral zeta function of the non-commutative harmonic oscillators. Ramanujan J. 15, 31-36 (2008)

A. Parmeggiani, Spectral Theory of Non-Commutative Harmonic Oscillators: An Introduction, Lecture Notes in Mathematics, vol. 1992 (Springer, Berlin, 2010)

A. Parmeggiani, M. Wakayama, Oscillator representations and systems of ordinary differential equations. Proc. Nat'1. Acad. Sci. USA 98, 26-30 (2001)

C. Reyes-Bustos, M. Wakayama, Spectral degeneracies in the asymmetric quantum Rabi model, in Mathematical Modelling for Next-Generation Cryptography, eds. by T. Takagi et al., Mathematics for Industry, vol. 29 (Springer, Berlin, 2017), pp. 117-137

C. Reyes-Bustos, M. Wakayama, The heat kernel and spectral zeta function for the quantum Rabi model, arXiv:1906.09597

F. Rodriguez-Villegas, Modular Mahler Measures I, Topics in Number Theory (Kluwer, Berlin, 1999), pp. 17-48

S. Sugiyama, Spectral zeta functions for the quantum Rabi models. Nagoya Math. J. 229, 52-98 (2018)

M. Wakayama, Equivalence between the eigenvalue problem of non-commutative harmonic oscillators and existence of holomorphic solutions of Heun differential equations, eigenstates degeneration and the Rabi model. Int. Math. Res. Notices 2016(3), 759-794 (2016)

M. Wakayama, Symmetry of asymmetric quantum Rabi models. J. Phys. A: Math. Theor. 50, 174001 (2017) 
F. Yoshihara et al., Inversion of Qubit Energy Levels in Qubit-Oscillator Circuits in the DeepStrong-Coupling Regime. Phys. Rev. Lett. 120, 183601 (2018)

D. Zagier, Integral solutions of Apéry-like recurrence equations, in Groups and Symmetries, CRM Proceedings \& Lecture Notes, vol. 47 (American Mathematical Society, Providence, 2009), pp. 349-366

Open Access This chapter is licensed under the terms of the Creative Commons Attribution 4.0 International License (http://creativecommons.org/licenses/by/4.0/), which permits use, sharing, adaptation, distribution and reproduction in any medium or format, as long as you give appropriate credit to the original author(s) and the source, provide a link to the Creative Commons license and indicate if changes were made.

The images or other third party material in this chapter are included in the chapter's Creative Commons license, unless indicated otherwise in a credit line to the material. If material is not included in the chapter's Creative Commons license and your intended use is not permitted by statutory regulation or exceeds the permitted use, you will need to obtain permission directly from the copyright holder.

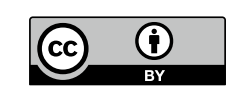

